\title{
In situ observation of degradation of electrocatalysts in humidified air atmosphere using a cold FE 60-300 kV ETEM
}

\author{
Manabu Shirai ${ }^{1}$, Hiroaki Matsumoto ${ }^{1}$, Isao Nagaoki ${ }^{2}$, ToshieYaguchi ${ }^{2}$, Takahiro Shimizu ${ }^{3}$ and Takeo \\ Kamino $^{3}$ \\ ${ }^{1}$ Application Development Department, Science System Design Division, Hitachi High-Technologies \\ Corporation, Hitachinaka, Ibaraki 312-0033, Japan \\ ${ }^{2}$ Electron Microscope Systems Design 2nd Department, Science System Design Division, Hitachi High- \\ Technologies, Hitachinaka, Ibaraki, 312-8504, Japan \\ 3. Japan Automobile Research Institute, 2530 Karima, Tsukuba, 305-0822 Japan
}

Polymer electrolyte fuel cells (PEFCs) attract a great attention as new generation power devices for automobile and cogeneration systems. Yet, the durability of electrocatalysts is one of the most critical issues that are delaying the commercialization of this technology. To improve the durability PEFCs, we focus on studying the degradation mechanism of the electrocatalysts in their real-time evolution during operation. For this purpose, in situ observation using an environmental transmission electron microscope (ETEM) is one of the most powerful techniques. We have developed a CFE-ETEM with capabilities of simultaneous SEM with STEM image observation and applied it to the study of Pt/CB electrocatalysts degradation mechanism [1]. Previous study revealed that the Pt particles on the carbon support were aggregated and the carbon support was depressed by the Pt particles under a dry air atmosphere at $200{ }^{\circ} \mathrm{C}$. However, the mechanism of the particles penetration into the carbon support is an open question. In the present study, we have investigated the behavior of Pt particles and related corrosion of the carbon support of electrocatalysts in a highly humidified air atmosphere.

In situ simultaneous SEM/STEM study of Pt/VC (Vulcan XC-72 carbon) electrocatalysts was performed using a Hitachi HF-3300 TEM combined with a newly developed humidified air supply system [2]. Figure 1 shows an external view of the humidified air system. This system consists of a humidifier, a duct hose and a built-in thermo-hygrometer. The humidified air from the humidifier is introduced into the specimen chamber of the microscope via a gas injection nozzle of a specimen holder. In situ observation in a humidified air atmosphere was carried out with the gas injection specimen heating holder [3]. Figure 2 shows an external view (a) and a schematic illustration of the specimen heating holder (b). The heating element is a spiral-shaped tungsten wire with $50 \mu \mathrm{m}$ in diameter and the electrocatalysts specimens are directly mounted on the wire using a small paintbrush. We observed structural change of the $\mathrm{Pt} / \mathrm{VC}$ electrocatalysts at $200{ }^{\circ} \mathrm{C}$ under the humidified air. The relative humidity and temperature of the introduced air was $99 \%$ and $40{ }^{\circ} \mathrm{C}$, respectively.

Figure 3 shows a sequence of SEM and dark-field (DF) STEM images observed at $200{ }^{\circ} \mathrm{C}$ during the humidified air injection. After 60 seconds in $2.4 \times 10^{-3} \mathrm{~Pa}$, the $\mathrm{Pt}$ particles made a movement and the agglomeration of the Pt particles have occurred on the carbon support. After 120 seconds in $2.4 \times 10^{-3} \mathrm{~Pa}$, the particles actively moved and the grain size increased significantly. After 600 seconds in $2.0 \times 10^{-2} \mathrm{~Pa}$, the number of $\mathrm{Pt}$ particles in the SEM image reduced drastically. This result suggested that the $\mathrm{Pt}$ particles depressed and the particles inserted into the carbon support. These experiments demonstrated that this instrument allows for in situ observation under a highly humidified air atmosphere. Furthermore, it is evident that the simultaneous dynamic observation of SEM and STEM images is indispensable to analyze structural change of nanomaterials. 
References:

[1] H. Matsumoto et al., Microscopy and Analysis 27(7), 13-18 (2013)

[2] T. Yaguchi et al., J. Electron Microsc. 61, 199-206 (2012)

[3] T. Kamino et al., J. Electron Microsc. 54(6), 497-503, (2005)

[4]The authors acknowledge to Prof. K. Sasaki and associate Prof. A. Hayashi of Kyushu University for providing the electrocatalyst samples and giving their fruitful comments.

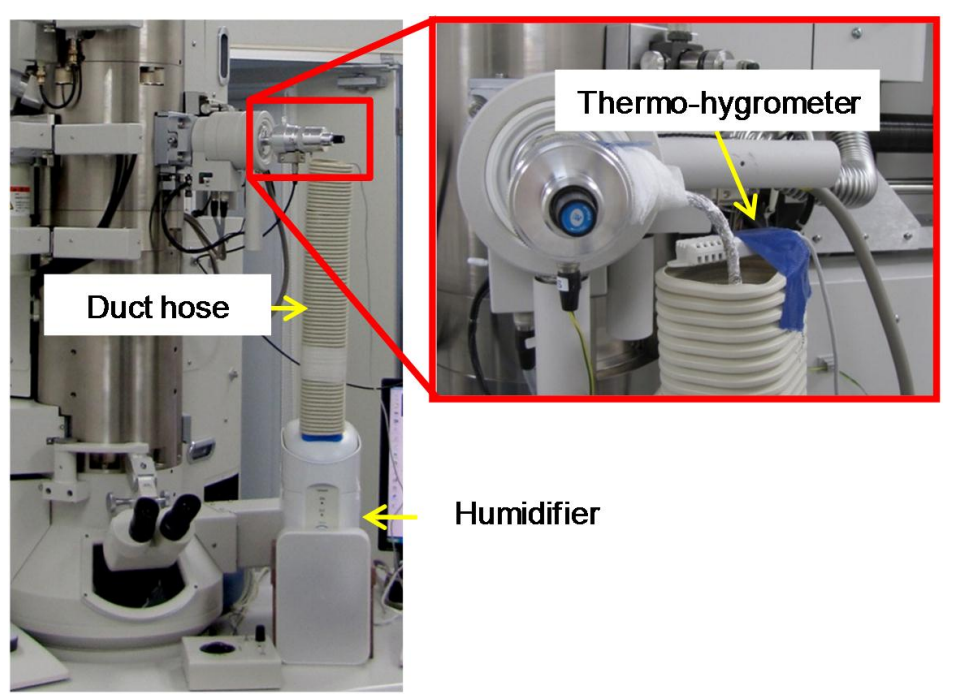

Figure 1. External view of a humidified air supply system developed for the HF-3300 Cold-FE TEM. (a)

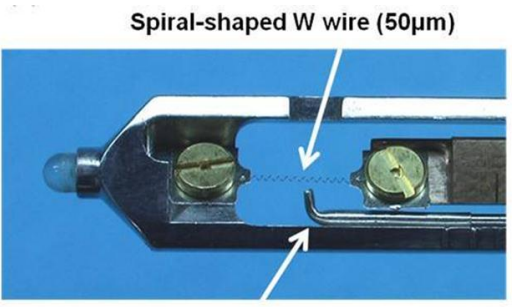

Gas injection nozzle, $0.5 \mathrm{~mm} \Phi$, $<1 \mathrm{~mm}$ from the heater

(b)

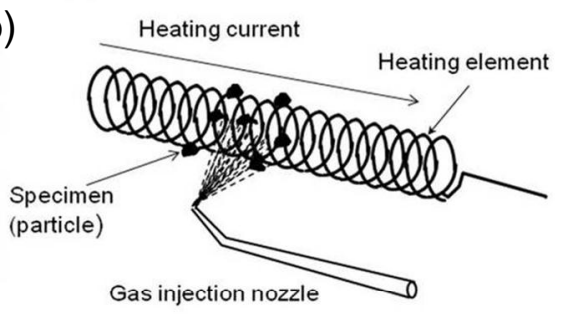

Figure 2. Gas injection specimen heating holder (a) and its schematic illustration (b).

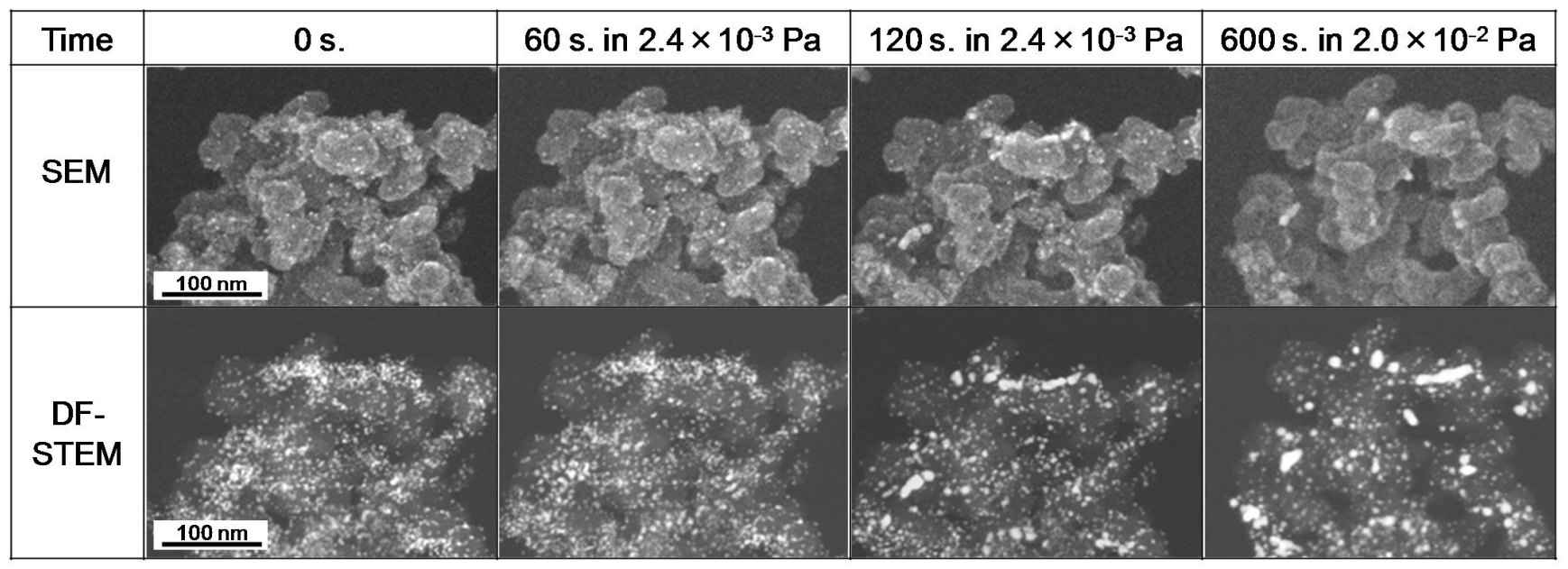

Figure 3. Sequence of SEM and DF-STEM images recorded at $200{ }^{\circ} \mathrm{C}$ in a humidified air atmosphere. 